\title{
Optimal Multi-Criteria Design of Hybrid Power Generation Systems: A New Contribution
}

\author{
M. Algabalawy \\ Elec. Maintenance \\ Engineer, \\ General Motors \\ Egypt
}

\author{
S.F. Mekhamer \\ Elec. Power and \\ Machines Dep. \\ Ain Shams \\ University, \\ Abbassia, Cairo
}

\author{
A.Y. Abdelaziz \\ Elec. Power and \\ Machines Dep. \\ Ain Shams \\ University, \\ Abbassia, Cairo
}

\author{
M.A. L Bader \\ Elec. Power and \\ Machines Dep. \\ Ain Shams \\ University, \\ Abbassia, Cairo
}

\begin{abstract}
Continuous researching effort to improve the power systems quality problems and to meet the increasing power demand has being done. Hybrid power generation systems (HPGS) considered one from the suggested solutions; these systems are combined from different power sources. Wind turbine (WT), photovoltaic (PV), storage battery (SB), fuel cell (FC), and gas turbine (GT) considered the most common power sources and the main elements of HPGS. There are two main modes for the HPGS; a stand-alone mode and the utility connected mode. This paper presents the optimal design of the HPGS for the two modes, where each mode is discussed for two scenarios, one for the winter and the other for the summer. Modern Meta-heuristic optimizations techniques have been used to keep the results effectiveness and efficiency. Moth-flame optimization (MFO) and multi-verse optimization (MVO) have been used to obtain the optimal design and sizing of the above mentioned power sources. This paper also presents a detailed comparison between the two scenarios for each mode, considering the total annual cost, emission, which are the most important common factors for the detailed comparison.
\end{abstract}

\section{Keywords}

Hybrid generation system, multi-criteria design, renewable energy sources, distributed generation, moth-flame optimization, multi-verse optimization, flower pollination algorithm.

\section{INTRODUCTION}

Electricity considered the backbone of any development and sophistication of the new societies. Increasing the demand is the complex issue, which facing the electrical company responsible and government. Blackout, electricity shortage, and power quality problems are the normal reactions of demand increasing without meeting this demand increasing. The solutions for these problems considered the most important challenges for the government. Researchers interest to share the responsibility with their government and present the new solutions to abort these problems as possible as much. Micro-grid, hybrid power generation system (HPGS), and distribution generation concepts considered the most accepted for the above-mentioned problems. Much of researchers go on improving and modifying these solutions to be applicable for other applications. HPGS occupies the largest interest of many researchers, where they state that, these systems could be installed near to the load; this means that, the transmissions line power losses would be minimized. HPGS includes wind turbine (WT), photovoltaic (PV), and storage battery (SB). Gas turbine (GT) and fuel cell (FC) are other power sources may be included to the pervious power sources. These sources considered the most prominent ones, the combinations of these sources are termed as HPGS. These combinations operate at two common modes; stand-alone mode, and utility connected mode. In this paper we will study the two modes in detailed, where each mode would be discussed for two scenarios; for the winter and the summer. We will use the existing natural gas distribution network to feed the GT by the natural gas as a fuel, considering all conditions of this network such as the flow velocity and the pressure drop. Meta-heuristic techniques have been used to obtain the optimal sizing of the HPGS for each mode. Moth-flame optimization (MFO) and multi-verse optimization (MVO) techniques considered from the most modern ones, and these techniques used for the first time in this application.

In reference, [1], L. Wang and C. Singh design a hybrid power generation system including wind power and solar power based on cost, reliability, and emission criteria. In addition, they consider the system uncertainties, such as equipment failures and stochastic generation/load variations, are by conducting adequacy evaluation based on probabilistic methods. They apply the modified particle swarm optimization to obtain the hybrid system design for different scenarios.

L. Liqun and L. Chunxia, reference, [2], study the feasibility analysis of a wind-PV-battery system for an off-grid power station specially located in remote village of Dongwangsha, Shanghai. They build the simulation model and obtain the results for the proposed hybrid WT-PV system with SB backup. They discuss the greenhouse gases (GHG) emissions, project costs and savings/income summary, financial viability, and risk analysis. They consider the proposed hybrid system is more environmental friendly as compared with the diesel only system.

In reference [3], S. L. Trazouei, F. L. Tarazouei, and M. Ghiamy present a design of a stand-alone hybrid WT/PV/diesel power generation system using imperialist competitive algorithm (ICA), particle swarm optimization (PSO) and ant colony optimization (ACO). They try to minimize of annual cost function of hybrid system considering the reliability and loss of power probability (LPSP) reliability index. Finally, they present the results; number of PV panels, number of WT, capacity of SB, total annual cost, and power diagram of hybrid power system components and reliability diagram for WT/PV/diesel hybrid systems.

In reference, [4], T. Tahri, A. Bettahar, and M. Douanitry to design the WT/PV/diesel hybrid power system for a village of AinMerane, Chlef, Algeria, considering the wind speed and solar radiation measurements. They apply the HOMER 
software to optimize a hybrid WT/PV/diesel system in term of technical and economic feasibility. They make a comparison between the performance of WT/PV/diesel system and the $\mathrm{WT} / \mathrm{PV} /$ diesel connecting to the utility grid system.

In reference, [5], Q. A. J. Jawad1, K. K. Gasem, and M. R. Jawad design and simulate a WT/PV/diesel hybrid power system (HPS) for the far areas of their country. They compare the proposed connecting configurations to select the one with the best efficiency of power consumption to the consumers by considering each power sources independently. It found as they consider that, the best efficiency of power consumption achieved with the Mixed-coupling HPSs, when compared with the other topologies and the selected topology used for further investigation.

T. Jima, reference, [6], presents the design of a hybrid electric power generation system utilizing both wind and solar energy for supplying a cluster of three micro and small enterprises (MSEs) working on wood and metal products at the Welenchity site. The potential energy of wind and solar sources for the desired site investigated, where he collects the wind speed and solar irradiation data for the site understudy. He uses the HOMER software to design the standalone PVWT/SB/diesel hybrid power system. Annual system cost has been minimized to the number of PV array number, WT swept area, SB capacity, and the required capacity of the diesel.

A.Bhowmik, reference, [7], apply the Microsoft excel software programming package to analyze the data measurements of all the components of the system. He uses MATLAB software package to develop the simulation program for proper optimization of the cost. A comparison has been done to ensure that the accuracy of the results. Lastly, he apply the 'observe and focus' algorithm to get more effective data, hence, to enhance the optimization of the hybrid system.

In reference, [8], M. Sekar, S. Arunkumar, and V. Balasubramanian describe an analysis of local PV-WT hybrid systems for supplying electricity to a private house, farmhouse or a small company with electrical power depending on the need at the site. The PV-WT hybrid systems extensively used to illustrate electrical concepts in hands-on laboratories and demonstrations in the Industrial Technology curriculum.

H. M. Farghally, F. H. Fahmy, and M. A. H.EL-Sayed, reference, [9], present the sizing of complete PV-WT hybrid systems for supplying electricity to emergency hospital, school and home buildings according to their energy requirements. They use the HOMER software to solve the optimization problem to minimize the objective function considering the different constraints and provide the size of PV-WT hybrid systems. In addition, they develop a neural network controller for achieving the coordination between system components as well as control the energy flows.

In reference, [10], P. Gajbhiye, and P. Suhane propose a hybrid energy system (HES) which combines PV and WT as a small-scale alternative source of electrical energy for the problem of sizing and economic assessment such that the demand of residential area is met. They use diesel generator (DG) and battery bank to cover the emergency loads energy, where covering the load demand under varying weather conditions. They consider all costs like capital cost, replacement cost, operation and maintenance cost and fuel cost. They claim that, the proposed study would be feasible solution for distributed generation of electric power for standalone applications at remote locations.

H. Belmili, M. Haddadi, S. Bacha, and M. F. Almi, reference, [11], propose a sizing method of stand-alone PV-WT hybrid systems basing on techno-economic analysis and using objectoriented programming. This program is building around fundamentals photovoltaic and wind generators models, storage capacity model, loss of power supply probability (LPSP) algorithm. In addition, they show that, the proposed techno-economic algorithm to determine the system that would guarantee is liable energy supply with the lowest investment.

In reference, [12], A.M. Eltamaly and M. A. Mohamed introduce a design and simulation program for autonomous hybrid PV/wind/battery energy system. They determine the size of each component of the hybrid energy system for the lowest price of $\mathrm{kWh}$ generated and the best loss of load probability at highest reliability. They collect the required data such as; hourly wind speed, hourly radiation, and hourly load power. They use HOMER software to study the changing of the penetration ratio of wind/PV with certain increments. They calculate the required size of all components and the battery size to get the acceptable probability.

In reference, [13], D. M. Atia, F. H. Fahmy, N. M. Ahmed, and H. T. Dorrah determine the design for renewable energy system powered an aquaculture pond. They analyze the feasibility of the standalone and utility connected system using HOMER software, which determines whether renewable energy resources satisfy hourly electric demand or not. They compare the demand for the electrical energy for each hour of the year with the energy supplied by the system for that hour and calculate the relevant energy flow for each component in the model. They take into the consideration all system parameters such as; wind speed, solar irradiance, interest rate and capacity shortage. They claim that, the simulation results indicate that the hybrid system is the best choice in this study, yielding lower net present cost

A. Maleki and A. Askarzadeh, in reference, [14], recommend a framework to size a hybrid energy system based on PV, WT and FC. For this aim, they model all the components and define an objective function based on the total annual cost. They show that, in the optimization problem, the maximum allowable loss of power supply probability $\left(\mathbf{L P S P}_{\max }\right)$ is also considered to have a reliable system. In order to minimize the objective function, they apply the bee swarm optimization (ABSO) algorithm. Their simulation results show that, the $\mathrm{PV} / \mathrm{WT} / \mathrm{FC}$ is the most cost-effective hybrid system and WT/FC and PV/FC systems are in the other ranks, where they set the $\mathbf{L P S P}_{\text {max }}$ by $0 \%$ as a constraint.

In reference, [15], O. H. Mohammed, Y. Amirat, M. Benbouzid, and A.A. Elbaset present design a stand-alone hybrid PV and FC hybrid system without battery storage to supply the electric load demand of the city of Brest, Western Brittany in France. They show that, the proposed design is focused on economical performances and is mainly based on the loss of the power supply probability concept and based on the simulation model developed using HOMER software.

M. S. Alam, reference, [16], proposes a hybrid distributed power generation (DG) system consists of WT and FC. Worth mentioning that, he uses the fuzzy logic controller for power management in optimally way as his claim. He applies this technique in the belief of providing the required power to a residential load on a continuous basis based on the feasibility 
of economic power generation. Where, this controller directs power to a fixed voltage bus in the power-conditioning unit (PCU). The fixed voltage bus supplies the load, while the excess power is directed to the energy storage bank first and then to an electrolyzer, which is used to generate hydrogen for the fuel cell. He Completes system modeling and simulation using the HOMER software, and his hybrid controller has been simulated in Simulink/MATLAB environment.

B. Tudu, K. K. MandaI, and N. Chakraborty, in reference, [17], present the design and the sizing of a grid independent hybrid energy system consisting of micro hydro, PV, WT and FC for supplying a specific load. They apply the bees' algorithm (BA) technique to sizing of the hybrid system, and this technique is compared with the particle swarm optimization (PSO) and the system performance is evaluated in terms of the cost of the system. They consider the net present value in the belief of obtaining the optimal sizing. The system is designed, where the maximum utilization of the renewable resources to reduce the system carbon. In addition, apart from renewable resources feeds the FC electrolyser. They show that the initiated system is quite feasible in meeting the load and in terms of cost of energy and the both algorithms are capable of giving global solution, but PSO is fast in reaching optimal solution and takes less CPU time as compared to BA.

A new contribution of a hybrid power generation is presented in this paper, where the natural gas distribution network is involved in the concept of the HPGS for the first time, and it is used to supply the natural gas (fuel) to the gas turbine (GT). All operational conditions of it have been considered through the HPGS design, where there is no need a new investment for increasing the capacity of the natural gas distribution network. Therefore, this configuration is considered a new one where the natural gas distribution network is used for the first time as a partner of the HPGS. The optimal design is obtained using new meta-heuristic optimization techniques such as moth-flame optimization (MFO) and multi-verse optimization (MVO). Actually it should that, the MFO and MVO are used for the first time in the design of HPGS. MATLAB software is applied to obtain the optimal results of the HPGS design.

\section{PROBLEM FORMULAION [1]}

Two different combinations of the HPGS have been discussed in this paper. The first combination is termed as a stand-alone HPGS, where it consists of WT, PV, SB, and GT. The second is termed as utility connected HPGS, where it combines WT, PV, SB, GT, and the utility. Figure 1 shows the standalone HPGS and Figure shows 2 the utility connected HPGS. The main objective functions of the aforementioned HPGS are the total annual cost (TAC) and the system pollution (SP), and the main target of this paper is to minimize these functions.

$f=\min (T A C, S P)$

\section{Total Annual Cost (TAC)}

The TAC for the standalone HPGS is described as shown in equation 1 , while equation 2 describes the TAC for the utility connected mode.

$T A C=\frac{\sum_{i=W, P V, S B}\left(I_{i}-S_{P_{i}}+O M_{P_{i}}\right)}{N_{P}}+A F C_{N G}$

where,

$W, P V, S B$ indicate the wind power, solar power, and battery storage, respectively,

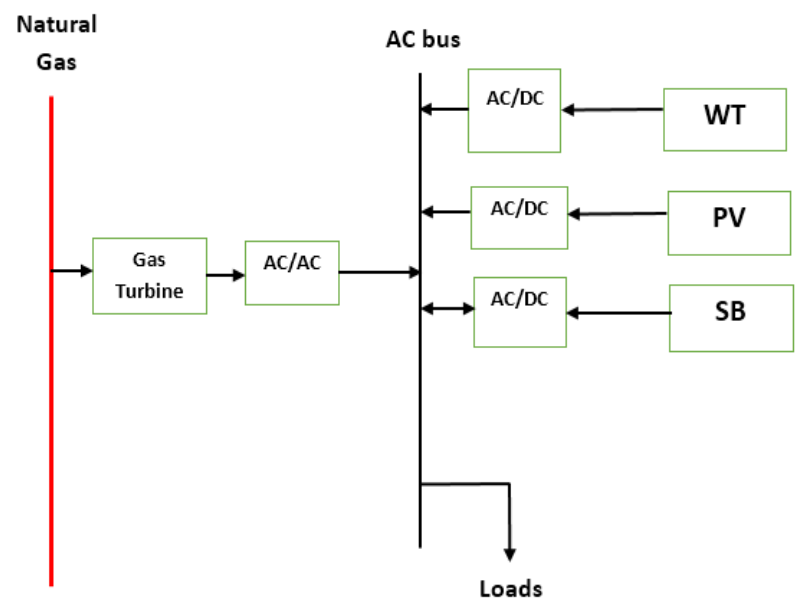

Fig. 1 Configuration of a Stand-Alone HPGS

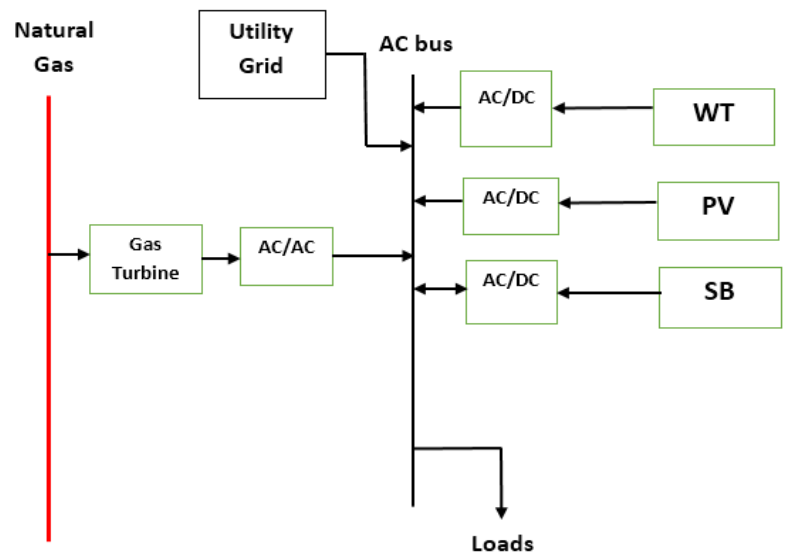

Fig. 2 Configuration of a Utility Connected HPGS

$I_{i}, S_{P_{i}}$, and $O M_{P_{i}}$ are the initial cost, present worth of salvage value, and present worth of operation and maintenance cost (OM) for equipment $i$, respectively.

$N_{P}$ is the lifespan of the project.

$A F C_{N G}$ is the annual purchasing natural gas cost from the NG distribution company.

$T A C=\frac{\sum_{i=W, P V, S B}\left(I_{i}-S_{P_{i}}+O M_{P_{i}}\right)}{N_{P}}+A F C_{N G}+A P P_{U T}$

where,

$A P P_{U T}$ is the annual purchased electric power from the utility grid in $(\$ / \mathrm{kW})$.

While the total system pollution (SP) of the standalone system per year is described as in equation 3 .

$S P=k_{1}+k_{2} \times \sum_{t=1}^{T} P_{G T, t}(t)+k_{3} \times\left(\sum_{t=1}^{T}\left(P_{G T, t}(t)\right)^{2}\right.$

Where only the gas turbine is the source of the pollution in this system. But the pollution sources of the utility connected system are the GT and the utility. Equation 4 shows the total pollution of the utility connected HPGS.

$S P=k_{1}+k_{2} \times \sum_{t=1}^{T}\left(P_{U T, t}(t)+P_{G T, t}(t)\right)+k_{3} \times$

$\left(\sum_{t=1}^{T}\left(P_{U T, t}(t)+P_{G T, t}(t)\right)^{2}\right.$

where,

$k_{1}, k_{2}$ and $k_{3}$ are the coefficients approximating the generator emission characteristics. 
$P_{G T, t}$ is the output power of the gas turbine at instant $t$ in $\mathrm{kW}$

$P_{U T, t}$ is the delivered power from the utility grid at instant $t$ in $\mathrm{kW}$.

The following sections illustrate the remind terms of equations 1 and 2 .

\section{A. TAC calculations \\ a- For wind turbine \\ 1- Initial cost \\ $I_{w}=\alpha_{w} A_{w}$ \\ where,}

$\alpha_{w}$ is the initial cost of wind turbine $\left(\$ / m^{2}\right)$

$A_{w}$ is the swept area $\left(m^{2}\right)$

2- Salvage value

$S_{P_{w}}=S_{w} A_{w}\left(\frac{1+\beta}{1+\gamma}\right)^{N_{P}}$

where,

$S_{w}$ is the salvage value of wind turbine $\left(\$ / \mathrm{m}^{2}\right)$

$\beta$ is the inflation rate

$\gamma$ is the interest rate

3- Operation and Maintenance cost

$O M_{P_{w}}=\alpha_{O M_{w}} A_{w} \sum_{i=1}^{N_{P}}\left(\frac{1+v}{1+\gamma}\right)^{i}$

where,

$\alpha_{O M_{w}}$ is the operation and maintenance cost of wind turbine system $\left(\$ / m^{2}\right)$

$v$ is the escalation factor

$b$ - For PV panels

1- Initial cost

$I_{P V}=\alpha_{P V} A_{P V}$

where,

$\alpha_{P V}$ is the initial cost of PV system $\left(\$ / m^{2}\right)$

$A_{P V}$ is the swept area $\left(\mathrm{m}^{2}\right)$

2- Salvage value

$S_{P_{P V}}=S_{P V} A_{P V}\left(\frac{1+\beta}{1+\gamma}\right)^{N_{P}}$

where,

$S_{P V}$ is the salvage value of PV system $\left(\$ / \mathrm{m}^{2}\right)$

3- Operation and Maintenance cost

$O M_{P_{P V}}=\alpha_{O M_{P V}} A_{P V} \sum_{i=1}^{N_{P}}\left(\frac{1+v}{1+\gamma}\right)^{i}$

$\alpha_{O M_{P V}}$ is the operation and maintenance cost of PV system $\left(\$ / m^{2}\right)$

c-For Storage Battery system

1- Initial cost

$I_{b}=\alpha_{b} P_{b_{c a p}} \sum_{i=1}^{X_{b}}\left(\frac{1+v}{1+\beta}\right)^{(i-1) N_{b}}$

where,

$\alpha_{b}$ is the initial cost of storage battery system ( $\left.\$ / k W\right)$

$P_{b_{c a p}}$ is the storage battery capacity $(k W)$

$N_{b}$ is the storage battery lifespan

$X_{b}$ is the number of times to purchase the batteries during the project lifespan $N_{P}$.

2- Salvage value

There is no salvage value for the storage battery system

\section{3- Operation and Maintenance}

$O M_{P_{S B}}=\alpha_{O M_{S B}} P_{b_{c a p}} \sum_{i=1}^{N_{P}}\left(\frac{1+v}{1+\gamma}\right)^{i}$

where,

$\alpha_{O M_{S B}}$ is the operation and maintenance cost of storage battery system $(\$ / \mathrm{kW})$.

d- For Gas Turbine

1- Initial cost

$I_{G T}=\alpha_{G T} P_{c a p}{ }_{G T}$ where,

$\alpha_{G T}$ is the initial cost of gas turbine ( $\left.\$ / \mathrm{kW}\right)$

$P_{c a p_{G T}}$ is the gas turbine capacity $(\mathrm{kW})$

2- Salvage value

$S_{P_{G T}}=S_{G T} P_{c a p}\left(\frac{1+\beta}{1+\gamma}\right)^{N_{P}}$

where,

$S_{G T}$ is the salvage value of gas turbine $(\$ / \mathrm{kW})$

$\beta$ is the inflation rate

$\gamma$ is the interest rate

3- Operation and Maintenance cost

$O M_{P_{G T}}=\alpha_{O M_{G T}} P_{c a p} \sum_{G T}^{N_{P}}\left(\frac{1+v}{1+\gamma}\right)^{i}$

where,

$\alpha_{O M_{G T}}$ is the operation and maintenance cost of gas turbine system $(\$ / \mathrm{kW})$

$v$ is the escalation factor

Then the total initial cost is

$I_{t o t}=I_{W T}+I_{P V}+I_{S B}+I_{G T}$

, the total salvage value

$S_{P_{\text {tot }}}=S_{P_{W T}}+S_{P_{P V}}+S_{P_{G T}}$

, and the total operation and maintenance cost

$O M_{P_{\text {tot }}}=O M_{P_{W T}}+O M_{P_{P V}}+O M_{P_{S B}}+O M_{P_{G T}}$

$e$-The annual cost for purchasing natural gas

$A F C_{N G}=\sum_{t=1}^{8760} m \cdot(t) \times \varphi_{N G}$

where,

$m \cdot(t)$ is fuel rate in $m^{3} / h$ consumed by the gas turbine.

$\varphi_{N G}$ is the natural gas price $\$ / B T U$

All components of equation 1 are detailed as shown in equation 5 to 19 .

\section{$f$ - The annual cost for purchasing the electricity}

It is considered only for the second case of utility connected HPGS, and it is as follows:

$A P C_{U T}=\sum_{t=1}^{8760} P_{U T, t} \times \varphi_{P}$

where,

$P_{U T, t}$ is the power delivered from the utility grid at an instant $k W$

$\varphi_{P}$ is the power delivered from the utility grid price $\$ / k W$.

All components of equation 2 are detailed as shown in equation 5 to 20 .

\section{B. Design Constraints}

There are much of constraints should be satisfied throughout system operations for any feasible solution. 


\section{Power balance constraint}

For any period $t$, the total power supply from the hybrid generation system must supply the total demand $P_{d}(t)$ with a certain reliability criterion. This relation can be represented by:

$P_{w}(t)+P_{P V}(t)+P_{b}(t)+P_{G T}(t)+P_{U T}=P_{d}(t)$

Assuming that, the system loss is included into the $P_{d}(t)$.

where,

$P_{w_{i}}(t)=\left\{\begin{array}{cl}0 & V_{t}<V_{c i} \\ \frac{1}{2} \rho A_{w} V_{t}^{3} C_{p} & V_{c i} \leq V_{t}<V_{r} \\ P_{W T_{r}} & V_{r} \leq V_{t}<V_{c o} \\ 0 & V_{c o}<V_{t}\end{array}\right.$

$P_{w}(t)=P_{w_{i}}(t) \times A_{w} \times \eta_{w t}$

$P_{P V}(t)=H \times A_{P V} \times \eta_{P V}$

where,

$V_{t}$ is the wind speed $(\mathrm{m} / \mathrm{sec})$

$V_{c i}$ is the wind turbine cut-in speed $(\mathrm{m} / \mathrm{sec})$

$V_{r}$ is the wind turbine rated speed $(\mathrm{m} / \mathrm{sec})$

$V_{c o}$ is the wind turbine cut-off speed $(\mathrm{m} / \mathrm{sec})$

$P_{W T_{r}}$ is the rated power of the wind turbine $(\mathrm{kW})$

$C_{p}$ is the power coefficient of the WT

$P_{G T}(t)=\eta_{\text {overall }} \times m \times H H V$

where,

$\eta_{\text {overall }}$ is the gas turbine and the alternator overall efficiency, $m$ is fuel rate in $m^{3} / h$,

$H H V$ is the high heat value in mega-joule per cubic meter $\mathrm{MJ} / \mathrm{m}^{3}$, and to convert from the $\mathrm{MJ} / \mathrm{h}$ to $\mathrm{kWh}$, equation 26 should be multiplied by 0.278 [11].

It could notice from the left hand side of equation 22 that, there are five parameters should be tuned to find the optimal design of the HPGS. Regarding to equation $24, A_{w}$ is the first required parameter and $A_{P V}$ is the second one according to equation 25 . The third one is $P_{b}(t)$, which is the instantaneous discharging/ charging power of the SB. From equation 26, the fuel rate $m$ is the fourth one. Finally, the instantaneous power delivered from the utility $P_{U T, t}$ is the last parameter. The following section shows the lower and the upper limits of the aforementioned parameters.

Bounds of Design Variables

$A_{w_{\min }} \leq A_{w} \leq A_{w_{\max }}$

where,

$A_{w}$ is the wind turbine swept area $\left(\mathrm{m}^{2}\right)$

$A_{P V_{\text {min }}} \leq A_{P V} \leq A_{P V_{\text {max }}}$

where,

$A_{P V}$ is the PV swept area $\left(\mathrm{m}^{2}\right)$

The state of charge (SOC) of storage batteries $P_{b_{s o c}}$ should not exceed the capacity of storage batteries $P_{b_{c a p}}$ and should be larger than the minimum permissible storage level $P_{b_{\text {min }}}$, the total SB capacity should not exceed the allowed storage capacity $P_{b_{c a p_{\max }}}$ and the hourly charge or discharge power $P_{b}$ should not exceed the hourly inverter capacity $P_{b_{\max }}$.

$P_{b_{\text {min }}} \leq P_{b_{\text {soc }}} \leq P_{b_{\text {cap }}}$

$0 \leq P_{b_{\text {cap }}} \leq P_{b_{\text {cap }} \text { max }}$

$P_{b} \leq P_{b_{\max }}$

$0<m<m_{\text {max }}$

Section 2.3 shows the investigation of $m_{\text {max }}$ and the motivations of using natural gas distribution network.

$P_{U T t_{\text {min }}}<P_{U T t}<P_{U T t_{\text {max }}}$

\section{Brief Description of Natural Gas Distribution Network} [18 and 19]

In the last three decades the Egyptian governments encourage The usage of natural gas and plan to feed all people by it to save the exporting of the LPG. In addition, the emissions produced from the natural gas are less than the produced from other fossil fuels. Figure 3 show a comparison between the emissions of the fossil fuels. It could be noticed that, the natural gas has the lowest emission level. Moreover, the emission of the fossil fuels is measured in kilogram $(\mathrm{Kg})$ of carbon oxide $\left(\mathrm{Co}_{2}\right)$ per gigajoule $(\mathrm{GJ})$. There are three mainly processes of the NG systems from the extraction to the distribution. These processes are; production, transmission, and distribution.

In this paper the distribution phase only has been studied to consider its conditions during the HPGS design. This phase contains the pressure reduction stations, metering stations, odorant stations, and cities internal distribution networks. The pressure reduction station mainly reduces the high-pressure (30-70 bar) to medium-pressure (4-7 bar). The metering stations are used to count the consumed natural gas. The odorant stations function is to add the odorant materials on the natural gas to make it is easy to smell and discover the natural gas if there is a gas leakage. The internal natural gas distribution network contains the medium pressure network, distributed pressure regulators, and the low-pressure networks (100 mbar). Finally, this process is considered the final process and it is responsible to deliver the natural gas to the customer (industrial, commercial, and domestic) with required pressure, cubic meters, and odorant level. Figure 4 shows a section from the distribution network for an urban area, where the purple line is the medium pressure line and points 1 shows the inlet point of the medium pressure line. While points 2 show the inlet points of the pressure regulators.

Figure 5 shows the monthly electrical and natural gas loads profiles, where the blue line refers to the electrical load profile, the black line represents the natural gas load, and the red line shows the average energy load (in Million British Thermal Unit). While Figure 6 shows the daily consumption profiles. And it is easy to note the following from Figure 5:

1- The natural gas (NG) consumption increases during the winter (Nov., Dec., Jan., Feb., March, and April or 11, $12,01,02,03$, and 04) months and decreases during the summer months (May, June, July, August, Sep., and Oct. or $05,06,07,08,09$, and 10).

2- The electric power (EP) consumption decreases during the winter months and increases during the summer months, which represents exactly the opposite for NG consumption.

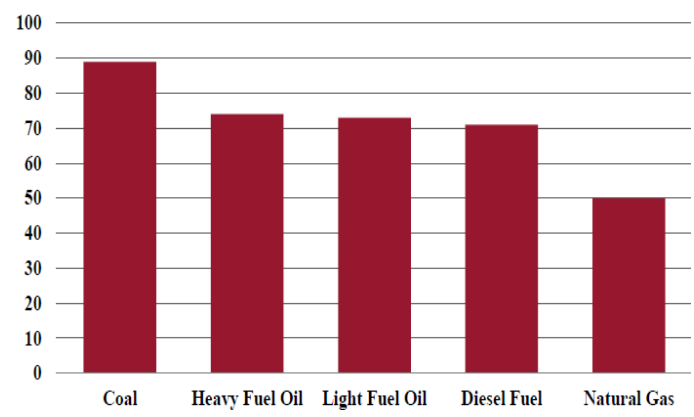

Fig. 3 Comparison of Fossil Fuels Emissions in $\mathrm{Kg} \mathrm{Co}_{2} / \mathrm{GJ}$ 


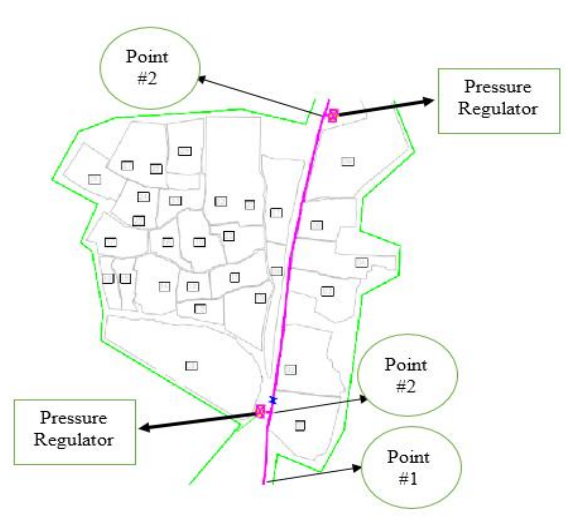

Fig. 4 the medium-pressure natural gas network of the urban area

From Figure 6 it could notice the following, where the thick line shows daily electrical load behavior and the thin line represents the daily natural gas load:

1- The off-peak periods of the natural gas load approximately synchronized with the on-peak periods of the electric power load, and vice versa. This means that, there is the ability to deliver natural gas to feed the fuel (NG) to the gas turbine without reducing the NG system reliability.

It is expected that; the instantaneous natural gas consumption is increased in case of the natural gas distribution network supplies the GT with required fuel this means the flow velocity increased in the natural gas pipes. If the flow velocity exceeds $20 \mathrm{~m} / \mathrm{s}$, the dust particles or debris will move, which these particles motion had the bad effect on the cooking devices, pressure regulators, and it may cause erosion to the internal surface of the pipeline. Equation 35 describes the flow velocity in $\mathrm{m} / \mathrm{sec}$.

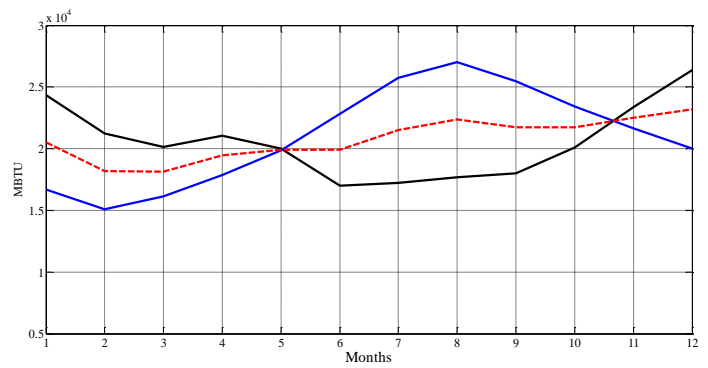

Fig. 5 Monthly consumption profiles (natural gas and electrical loads) of the studied area

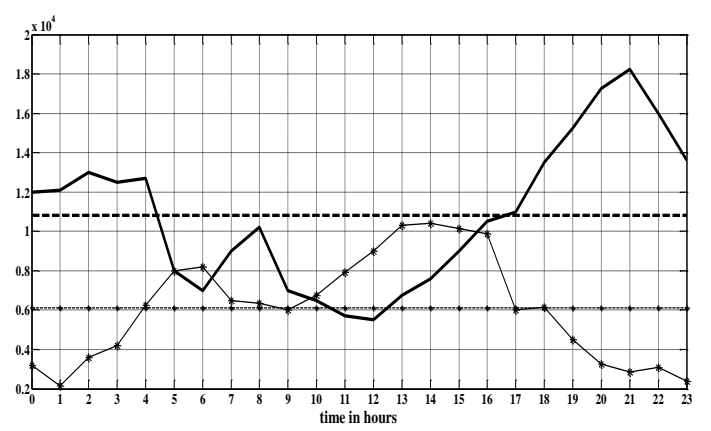

Fig. 6 the Daily consumption profiles (natural gas and electrical loads) of the studied area
$U=\frac{353 Q P_{b}}{d^{2}\left[P_{1}^{2}-\frac{3730 f L Q^{2}}{d^{5}}\right]^{0.5}}$

(34)

where,

$Q$ is the instantaneous flow of the natural gas

$P_{b}$ is the absolute pressure at datum conditions (bar) (atmospheric conditions)

$d$ is the internal diameter of the pipe $(\mathrm{mm})$

$f$ is the friction factor, and it is in the range of $(0.009$ to 0.015$)$

for corrugated Polyethylene (PE) pipes with smooth inner walls

$P_{1}$ is the absolute upstream (inlet) pressure (bar)

$L$ is the length of the pipe over which the velocity is being measured $(\mathrm{m})$.

Equation 38 shows the relationship between the regulator input pressure and the output capacity.

Regulator Capacity $=K_{G} \times \sqrt{P_{\text {out }}\left(P_{2}-P_{\text {out }}\right)}$

(35)

where,

$K_{G}$ is the sizing coefficient.

According to this equation, if the $P_{\text {out }}$ equals to $100 \mathrm{mbar}$, Regulator Capacity is required to equal to $1000 \mathrm{~m}^{3} / \mathrm{hr}$, the $P_{2}$ will be 0.458 bar in case of $K_{G}$ equals to 4200 . So, the minimum value of $P_{2}$ is 0.458 bar to keep the regulator capacity is $1000 \mathrm{~m}^{3} / \mathrm{hr}$. And if the equation 35 is solved at $U \leq 20 \mathrm{~m} / \mathrm{sec}$ and the $P_{2}$ is 0.458 , the maximum allowable capacity is $4793.48 \mathrm{~m}^{3} / \mathrm{hr}$. Therefore, the $m_{\text {max }}$ is 4793.48 .

\section{MOTH-FLAME OPTIMIZER (MFO)}

\section{[20]}

In this algorithm, it is assumed that the candidate solutions are moths and the problem's variables are the position of moths in the space. Therefore, the moths can fly in 1-D, 2-D, and 3-D with changing their position vectors. Since the MFO algorithm is a population-based algorithm. The moths are actual search agents that move around the search space, whereas flames are the best position of moths that obtains so far. In other words, flames considered as flags or pins that are dropped by moths when searching the search space. Therefore, each moth searches around a flag (flame) and updates it in case of finding a better solution. The algorithm author chose the logarithmic spiral as the main update mechanism of moths. However, any types of spiral can be utilized, where subject to the following conditions:

1- Spiral's initial point should start from the moth

2- Spiral's final point should be the position of the flame

3- Fluctuation of the range of spiral should not exceed from the search space

Considering these points, the logarithmic spiral for the MFO algorithm could be defined as follows:

$S\left(M_{i}, F_{i}\right)=D_{i} \cdot e^{b t} \cdot \cos (2 \pi t)+F_{i}$

where,

$D_{i}$ indicates the distance of the $i^{\text {th }}$ moth for the $j^{\text {th }}$ flame,

$b$ is a constant for defining the shape of the logarithmic spiral, and $t$ is a random number in $[-1,1]$.

$D$ is calculated as follows:

$D_{i}=\left|F_{i}-M_{i}\right|$

where,

$M_{i}$ indicate the $i^{t h}$ moth, 
$F_{j}$ indicates the $j^{\text {th }}$ flame, and

$D i$ indicates the distance of the $i^{t h}$ moth for the $j^{\text {th }}$ flame. Equations 36 and 37 simulate the spiral-flying path of moths Equation 37 shows the next position of a moth is defined with respect to a flame. The $t$ parameter in the spiral equation defines how much the next position of the moth should be close to the flame ( $t=-1$ is the closest position to the flame, while $t=1$ shows the farthest). Therefore, a hybrid ellipse around the flame in all directions and the next position of the moth can be assumed. Spiral movement considered the main component of the proposed method because it dictates how the moths update their positions around flames. The spiral equation allows a moth to fly "around" a flame and not necessarily in the space between them. Therefore, the exploration and exploitation of the search space can be guaranteed. The logarithmic spiral, space around the flame, and the position considering different $t$ on the curve are illustrated in Figures 7 and 8. The vertical axis shows only one dimension (1 variable/parameter of a given problem), but the proposed method can be utilized for changing all the variables of the problem. The possible positions (dashed black lines) that can be chosen as the next position of the moth (blue horizontal line) around the flame (green horizontal line) clearly show that a moth can explore and exploit the search space around the flame in one dimension. Exploration occurs when the next position is outside the space between the moth and flam as can be seen in the arrows labeled by 1, 3, and 4 . Exploitation happens when the next position lies inside the space between the moth and flame as can be observed in the arrow labeled by 2 . There are some interesting observations for this model as follow:

1- A moth can converge to any point in the neighborhood of the flame by changing $t$

2- The lower $t$, the closer distance to the flame.

3- The frequency of position updating on both sides of the flame is increased as the moth get closer to the flame

The proposed position updating procedure can guarantee the exploitation around the flames. In order to improve the probability of finding better solutions, we consider the best solutions obtained so far as the flames. Therefore, the matrix $\mathrm{F}$ in above equations always includes $n$ recent best solutions obtained so far. The moths are required to update their positions with respect to this matrix during optimization. In order to further emphasize exploitation, we assume that $t$ is a random number in $[r, 1]$ where $r$ is linearly decreased from -1 to -2 over the course of iteration. Note that we name $r$ as the convergence constant. With this method, moths tend to exploit their corresponding flames more accurately proportional to the number of iterations

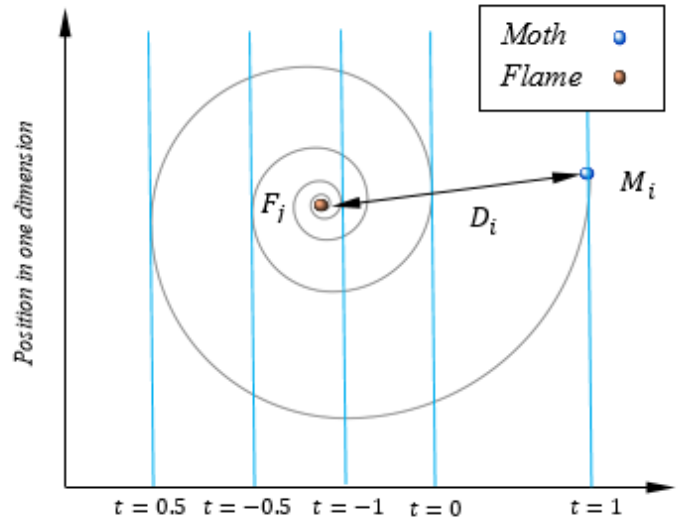

Fig. 7 The flame position at different $t$

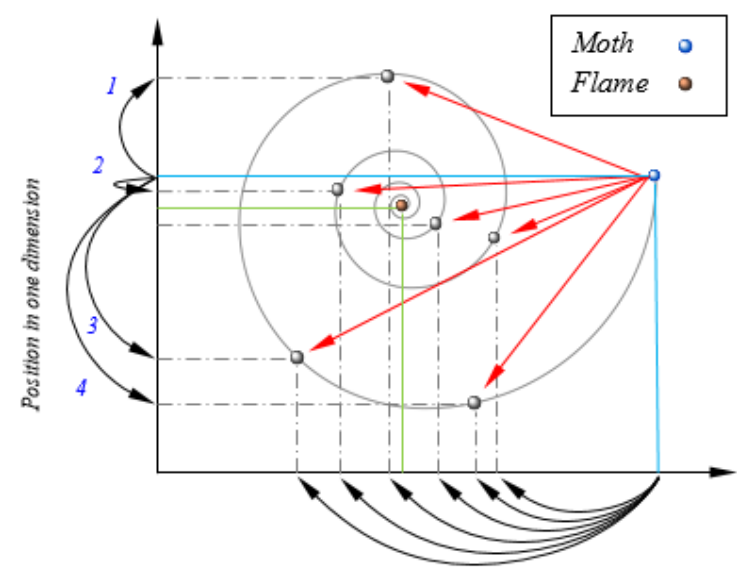

Fig. 8 the updated positions of the moth around the flame.

The flames are sorted based on their fitness values. The moths then update their positions with respect to their corresponding flames. The first moth always updates its position with respect to the best flame, whereas the last moth updates its position with respect to the worst flame in the list. It should be noted that this assumption is done for designing the MFO algorithm, while possibly it is not the actual behavior of moths in nature. However, the transverse orientation is still done by the artificial moths. If all of the moths get attracted to a single flame, all of them converge to a point in the search spaces because they can only fly towards a flame and not outwards. Requiring them to move around different flames, however, causes higher exploration of the search space and lower probability of local optima stagnation.

The exploration of the search space around the best locations obtained so far is guaranteed with this method due to the following reasons:

1- Moths update their positions in hybrid spheres around the best solutions obtained so far.

2- The sequence of flames is changed based on the best solutions in each iteration, and the moths are required to update their positions with respect to the updated flames. Therefore, the position updating of moths may occur around different flames, a mechanism that causes sudden movement of moths in search space and promotes exploration.

The position updating of moths with respect to $n$ different locations in the search space may degrade the exploitation of 
the best promising solutions. To resolve this concern, it is proposed that, an adaptive mechanism for the number of flames. The following formula has been used to update the number of flames over the course odd iterations:

flame no. $=\operatorname{round}\left(N-l * \frac{N-1}{T}\right)$

where,

$l$ is the current number of iteration,

$N$ is the maximum number of flames, and

$T$ indicates the maximum number of iterations.

The preceding figure shows that there is $N$ number of flames in the initial steps of iterations. However, the moths update their positions only with respect to the best flame in the final steps of iterations. The gradual decrement in number of flames balances exploration and exploitation of the search space.

After all, the general steps of the proposed MFO algorithm in one iteration are presented as follows:

Moth-Flame algorithm pseudo code

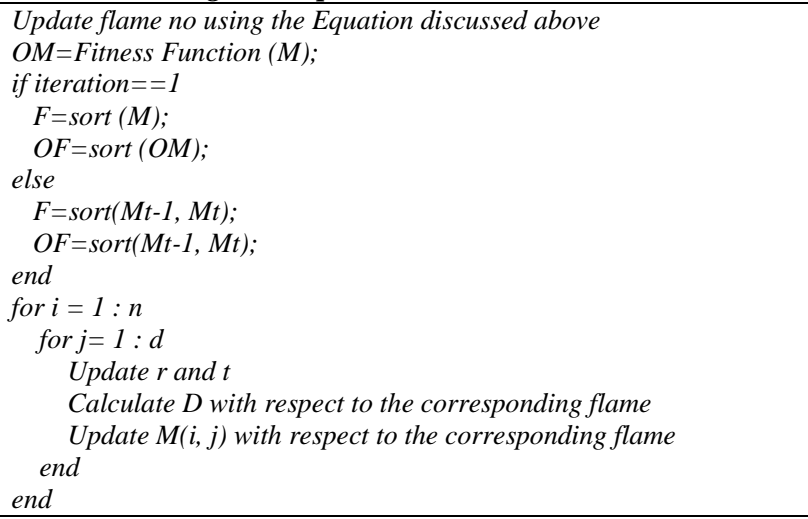

\section{MULTI-VERSE OPTIMIZER (MVO)} [21]

This algorithm had been initiated by S. A. Mirjalili, S. M. Mirjalili, and A. Hatamlou. And they show that, the main inspirations of this algorithm are based on three concepts in cosmology: white hole, black hole, and wormhole. The mathematical models of these concepts are developed to perform exploration, exploitation, and local search, respectively. Figure 9 shows the Conceptual models of these three key components of the multi-verse theory.
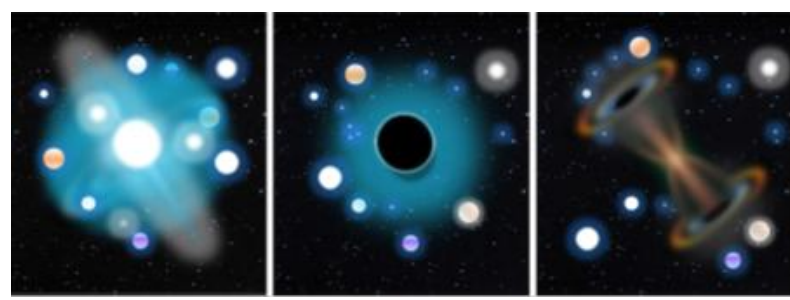

Fig. 9 White hole, black hole, and wormhole

The population-based algorithm divides the search process into two phases: exploration versus exploitation. The authors utilize the concepts of white hole and black hole in order to explore search spaces by MVO. In contrast, the wormholes assist MVO in exploiting the search spaces. They assume that each solution is analogous to a universe and each variable in the solution is an object in that universe. In addition, they assign each solution an inflation rate, which is proportional to the corresponding fitness function value of the solution. They also use the term time instead of the iteration since it is a common term in multi-verse theory and cosmology. During optimization, the following rules are applied to the universes of MVO:

1- The higher inflation rate, the higher probability of having white hole.

2- The higher inflation rate, the lower probability of having black holes.

3- Universes with higher inflation rate tend to send objects through white holes.

4- Universes with lower inflation rate tend to receive more objects through black holes.

5- The objects in all universes may face random movement towards the best universe via wormholes regardless of the inflation rate.

The conceptual model of the proposed algorithm is illustrated in Figure 10. This Figure shows that the objects are allowed to move between different universes through white/black hole tunnels. When a white/black tunnel is established between two universes, the universe with higher inflation rate is considered to have white hole, whereas the universe with less inflation rate is assumed to own black holes.

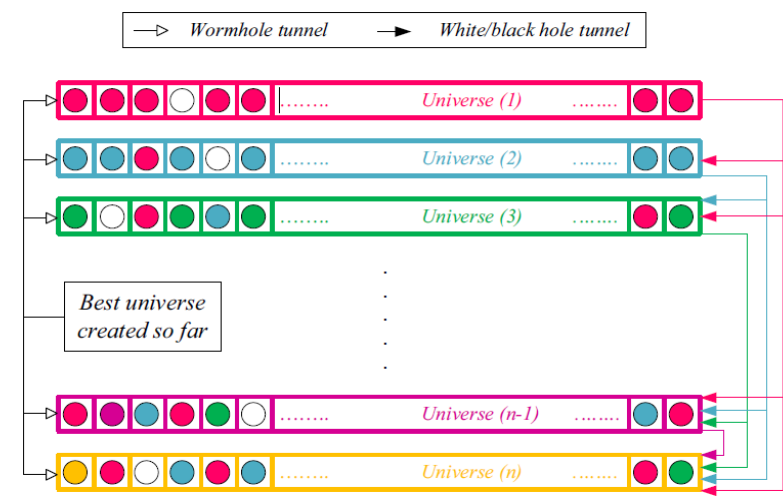

Fig. 10 Conceptual model of the proposed MVO algorithm

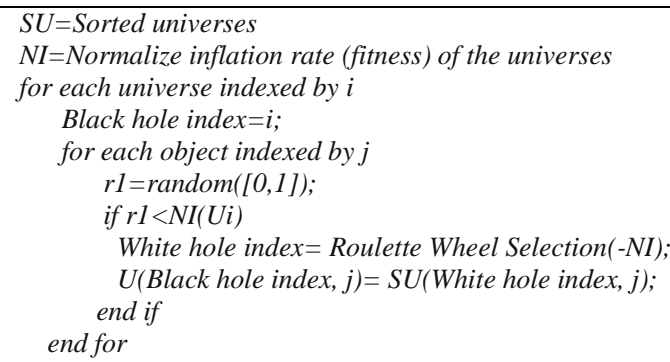

The objects are then transferred from the white holes of the source universe to black holes of the destination universe. This mechanism allows the universes to easily exchange objects. In order to improve the whole inflation rate of the universes, it is assumed that the universes with high inflation rates are highly probable to have white holes. In contrary, the universes with low inflation rates have a high probability of having black holes. Therefore, there is always high possibility to move objects from a universe with high inflation rate to a universe with low inflation rate. In order to mathematically model the white/black hole tunnels and exchange the objects of universes, the roulette wheel mechanism has been used. For each iteration, the universes based of their inflation rates are 
sorted and one of them is chosen by the roulette wheel to have a white hole. The following steps are done in order to do this.

Assume that

$U=\left[\begin{array}{cccc}x_{1}{ }^{1} & x_{1}{ }^{2} & \ldots & x_{1}{ }^{d} \\ x_{2}{ }^{1} & x_{2}{ }^{2} & \ldots & x_{2}{ }^{d} \\ \vdots & \vdots & \vdots & \vdots \\ x_{n}{ }^{1} & x_{n}{ }^{2} & \ldots & x_{n}{ }^{d}\end{array}\right]$

where,

$d$ is the number of parameters (variables) and

$n$ is the number of universes (candidate solutions):

$x_{i}^{j}= \begin{cases}x_{k}{ }^{j} & r 1<N I(U i) \\ x_{i}{ }^{j} & r 1 \geq N I(U i)\end{cases}$

where,

$x_{i}^{j}$ indicates the $j^{t h}$ parameter of $i^{t h}$ universe,

$U i$ shows the $i^{\text {th }}$ universe,

$N I(U i)$ is normalized inflation rate of the $i^{\text {th }}$ universe,

$r 1$ is a random number in $[0,1]$, and

$x_{k}{ }^{j}$ indicates the $j^{\text {th }}$ parameter of $k^{\text {th }}$ universe selected by a roulette wheel selection mechanism.

The selection and determination of white holes are done by the roulette wheel, which is based on the normalized inflation rate. The less inflation rate, the higher probability of sending objects though white/black hole tunnels. Please note that - NI should be changed to $N I$ for the maximization problems. The exploration can be guaranteed using this mechanism since the universes are required to exchange objects and face abrupt changes in order to explore the search space. With the above mechanism, the universes keep exchanging objects without perturbations. In order to maintain the diversity of universes and perform exploitation, we consider that each universe has wormholes to transport its objects through space randomly. In Figure 10, white points represent transferred objects through the wormholes. It may be observed that the wormholes randomly change the objects of the universes without consideration of their inflation rates. In order to provide local changes for each universe and have high probability of improving the inflation rate using wormholes, we assume that wormhole tunnels are always established between a universe and the best universe formed so far. The formulation of this mechanism is as follows:

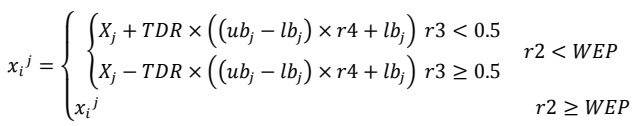

where,

$X_{j}$ indicates the $j^{t h}$ parameter of best universe formed so far,

$T D R$ is the travelling distance rate

$T D R=1-\frac{l^{1 / p}}{L^{1 / p}}$

$p$ defines the exploitation accuracy over the iterations, equal to 6

$l$ is the current iteration

$L$ is the maximum iterations

$W E P$ is the wormhole existence probability

$W E P=\operatorname{Min}+l \times\left(\frac{\text { Max-Min }}{L}\right)$

$\operatorname{Min}=0.2$
$\operatorname{Max}=1.0$,

$l b_{j}$ shows the lower bound of $j^{\text {th }}$ variable,

$u b_{j}$ is the upper bound of $j^{t h}$ variable,

$x_{i}{ }^{j}$ indicates the $j^{\text {th }}$ parameter of $i^{\text {th }}$ universe, and

$r 2, r 3$, and $r 4$ are random numbers in $[0,1]$.

The MVO pseudo code could be represented as follows:

Multi-Verse algorithm pseudo code

\section{APPLICATION TO A CASE STUDY} [1]

As was discussed, there are two different cases according to utility connecting or disconnecting. Each case would be discussed for different scenarios; one for winter and the other for the summer. MFO and MVO will be applied for these cases. Table 1 shows the required data.

Table 1 the used system parameters

\begin{tabular}{|l|c|c|}
\hline \multicolumn{1}{|c|}{ System parameters } & Values & Unit \\
\hline Wind turbine efficiency $\eta_{w t}$ & $50 \%$ & \\
\hline PV efficiency $\eta_{P V}$ & $16 \%$ & \\
\hline Storage battery efficiency $\eta_{S B}$ & $82 \%$ & \\
\hline Inflation rate $\beta$ & $9 \%$ & \\
\hline Interest rate $\gamma$ & $12 \%$ & \\
\hline Escalation rate $v$ & $12 \%$ & \\
\hline Lifespan of the project $N_{P}$ & 20 & year \\
\hline Lifespan of the wind turbine & 20 & year \\
\hline Lifespan of the PV & 20 & year \\
\hline Lifespan of the Storage battery & 10 & year \\
\hline Wind turbine initial cost $\alpha_{w}$ & 100 & $\$ / \mathrm{m} 2$ \\
\hline PV initial cost $\alpha_{P V}$ & 450 & $\$ / \mathrm{m} 2$ \\
\hline Storage Battery initial cost $\alpha_{b}$ & 100 & $\$ / \mathrm{kW}$ \\
\hline Wind turbine salvage value $S_{w}$ & 10 & $\$ / \mathrm{m}^{2}$ \\
\hline PV salvage value $S_{P V}$ & 45 & $\$ / \mathrm{m}^{2}$ \\
\hline O\&M cost of Wind turbine $\alpha_{O M_{w}}$ & 2.5 & $\$ / \mathrm{m}^{2}$ \\
\hline O\&M cost of PV $\alpha_{O M_{w}}$ & 4.3 & $\$ / \mathrm{m}^{2}$ \\
\hline O\&M cost of Storage Battery $\alpha_{O M_{w}}$ & 10.0 & $\$ / \mathrm{kW}$ \\
\hline Cut-in wind speed $V_{c i}$ & 2.5 & $\mathrm{~m} / \mathrm{s}$ \\
\hline Rated wind turbine speed $V_{r}$ & 12.5 & $\mathrm{~m} / \mathrm{s}$ \\
\hline Cut-out wind speed $V_{c o}$ & 20.0 & $\mathrm{~m} / \mathrm{s}$ \\
\hline Wind turbine rated power $P_{W T_{r}}$ & 4.0 & $\mathrm{~kW}$ \\
\hline Maximum swept area of wind turbine $A_{w}$ & 10000 & $\mathrm{~m}^{2}$ \\
\hline Minimum swept area of wind turbine $A_{w}$ & 400 & $\mathrm{~m}^{2}$ \\
\hline Maximum swept area of PV $A_{P V}$ & 8000 & $\mathrm{~m}^{2}$ \\
\hline Minimum swept area of wind turbine $A_{P V}$ & 200 & $\mathrm{~m}^{2}$ \\
\hline Maximum conversion capacity $P_{b_{m}}$ ax & 3 & $\mathrm{~kW}$ \\
\hline Minimum storage level $P_{b_{m i n}}$ & 3 & $\mathrm{~kW}$ \\
\hline Rated battery capacity $P_{b_{r}}$ & 8 & $\mathrm{~kW}$ \\
\hline Max total Storage Battery capacity $P_{b_{c a p}}$ & 40 & $\mathrm{~kW}$ \\
\hline Price of natural gas price $\varphi_{N G}$ & 0.082 & $\$ / \mathrm{m}^{3}$ \\
\hline & 4.09 & \\
\hline & -5.5 & \\
\hline & 6.5 & \\
\hline
\end{tabular}

Loads consumption, wind speed, solar irradiation, and the average natural gas consumptions are shown in table 2 . 
Table 2 Input data

\begin{tabular}{|l|c|c|}
\hline \multicolumn{1}{|c|}{ System parameters } & Sce. I & Sce. II \\
\hline Ave. power load $(\mathrm{kW})$ & 1500 & 2250 \\
\hline Ave. wind speed $(\mathrm{m} / \mathrm{s})$ & 5.3 & 4.87 \\
\hline $\begin{array}{l}\text { Ave. Solar irradiance }(\mathrm{W} / \\
\left.\mathrm{m}^{2}\right)\end{array}$ & 100.5 & 172.8 \\
\hline $\begin{array}{l}\text { Ave. natural gas load }\left(\mathrm{m}^{3} /\right. \\
\mathrm{hr})\end{array}$ & 850 & 645 \\
\hline
\end{tabular}

\section{RESULTS}

The most modern two optimization have been used for the first time in this applications to obtain the optimal design of the HPGS. MFO and MVO have been applied for this problem, and the obtained results have been compared to the obtained by applying flower pollination algorithm (FPA). The following four Tables $(3,4,5$, and 6) show the results of applying MFO, MVO, and FPA for the two different cases and each case for the two mentioned scenarios. Table 3 shows the results obtained of using MFO, MVO, and FPA for the stand-alone hybrid system at scenario I.

Table 3 the optimal results of a stand-alone HPGS at scenario I

\begin{tabular}{|c|c|c|c|}
\hline \multirow{2}{*}{ Variable } & \multicolumn{3}{|c|}{ A stand-alone system (Scenario I) } \\
\cline { 2 - 4 } & MFO & MVO & FPA \\
\hline$A_{w}\left(m^{2}\right)$ & 10000 & 10000 & 8700 \\
\hline$A_{P V}\left(m^{2}\right)$ & 161.38 & 1945 & 278 \\
\hline$P_{b_{c a p}}(\mathrm{~kW})$ & 1000 & 1000 & 1000 \\
\hline$P_{b}(\mathrm{~kW})$ & 100 & 100 & 100 \\
\hline$P_{G T_{c a p}}(\mathrm{~kW})$ & 1500 & 1500 & 1500 \\
\hline$m \cdot\left(\mathrm{m}^{3} / \mathrm{hr}\right)$ & 378 & 371.11 & 403 \\
\hline TAC $(\$ /$ year $)$ & 279,486 & 253,246 & 294,320 \\
\hline SP $($ ton/year $)$ & $1.3840 \mathrm{e}+11$ & $1.339 \mathrm{e}+11$ & $1.402 \mathrm{e}+11$ \\
\hline
\end{tabular}

For this table we observe that, the first two optimizations techniques (MFO and MVO) give the more superiority than using CSA and FPA. Where the annual total cost in case of applying MFO and MVO is lower than applying FPA. Moreover, the annual emission in case of applying MFO and MVO is lower than applying FPA.

Table 4 shows the obtained results of applying the above mentioned optimization techniques for the case of utility connected hybrid system at scenario I.

From table 4, it could be noted that, the MFO and MVO give the most superiority results, where the annual total cost in case of applying MFO and MVO is lower than applying FPA. Moreover, the annual emission in case of applying MFO and MVO is lower than applying FPA.

From Tables 3 and 4 we can observe the following for the two different cases (a stand-alone and utility connected) at scenario I:

1- The annual cost in case of applying MFO and MVO is lower than that, in case of applying FPA.

Table 4 the optimal results of utility connected HPGS at scenario I

\begin{tabular}{|c|c|c|c|}
\hline \multirow{2}{*}{ Variable } & \multicolumn{3}{|c|}{ Utility connected system (Scenario I) } \\
\cline { 2 - 4 } & MFO & MVO & FPA \\
\hline$A_{w}\left(m^{2}\right)$ & 736 & 1160 & 2078 \\
\hline$A_{P V}\left(m^{2}\right)$ & 2401 & 3620 & 3045 \\
\hline
\end{tabular}

\begin{tabular}{|c|c|c|c|}
\hline$P_{b_{c a p}}(\mathrm{~kW})$ & 1000 & 1000 & 1000 \\
\hline$P_{b}(\mathrm{~kW})$ & 100 & 100 & 100 \\
\hline$P_{G T_{c a p}}(\mathrm{~kW})$ & 1500 & 1500 & 1500 \\
\hline$P_{U T_{c a p}}(\mathrm{~kW})$ & 367.86 & 84 & 325 \\
\hline$m \cdot\left(m^{3} / \mathrm{hr}\right)$ & 315.6 & 384.5 & 305.6 \\
\hline TAC $(\$ /$ year $)$ & $376,851.6$ & 342,235 & 382,419 \\
\hline SP $($ ton/year $)$ & $1.5870 \mathrm{e}+11$ & $1.5867 \mathrm{e}+11$ & $1.5901 \mathrm{e}+11$ \\
\hline
\end{tabular}

2- The annual cost for the stand-alone HPGS is lower than that, for the utility connected HPGS.

3- The annual emission in case of applying MFO and MVO is lower than that, in case of applying FPA.

4- The annual emission for the stand-alone HPGS is lower than that, for the utility connected HPGS.

5- The natural gas consumption in case of applying MFO and MVO is lower than that, in case of applying FPA.

6- The natural gas consumption for the stand-alone HPGS is higher than that, for the utility connected HPGS

Table 5 shows the results obtained of using MFO, MVO, and FPA for the stand-alone hybrid system at scenario II.

Table 5 the optimal results of a stand-alone HPGS at scenario II

\begin{tabular}{|c|c|c|c|}
\hline \multirow{2}{*}{ Variable } & \multicolumn{3}{|c|}{ Utility connected system (Scenario I) } \\
\cline { 2 - 4 } & MFO & MVO & FPA \\
\hline$A_{w}\left(m^{2}\right)$ & 100 & 6729 & 5138 \\
\hline$A_{P V}\left(m^{2}\right)$ & 10000 & 8766 & 7583 \\
\hline$P_{b_{c a p}}(\mathrm{~kW})$ & 1000 & 1000 & 1000 \\
\hline$P_{b}(\mathrm{~kW})$ & 100 & 100 & 100 \\
\hline$P_{G T_{c a p}}(\mathrm{~kW})$ & 1500 & 1500 & 1500 \\
\hline$m \cdot\left(\mathrm{m}^{3} / \mathrm{hr}\right)$ & 478 & 463 & 435 \\
\hline TAC $(\$ /$ year $)$ & 466,041 & 427,671 & 489,612 \\
\hline SP $($ ton/year $)$ & $2.1033 \mathrm{e}+11$ & $2.0766 \mathrm{e}+11$ & $2.1052 \mathrm{e}+11$ \\
\hline
\end{tabular}

From table 5 we find that, MVO produces the most superiority results, where its annual cost and emission considered the lowest values. Generally, applying MFO and MVO gives annual cost and emission lower than applying FPA.

Table 6 shows the results obtained of using MFO, MVO, and FPA for the utility connected hybrid system at scenario II.

From table 6 we find that, MVO gives the most superiority results, where its annual cost and emission considered the lowest values.

From Tables 3 and 4 we can observe the following for the two different cases (a stand-alone and utility connected) at scenario II:

1- The annual cost in case of applying MFO and MVO is lower than that, in case of applying FPA.

2- The annual cost for the stand-alone HPGS is lower than that, for the utility connected HPGS.

3- The annual emission in case of applying MFO and MVO is lower than that, in case of applying FPA.

4- The annual emission for the stand-alone HPGS is lower than that, for the utility connected HPGS.

5- The natural gas consumption for the stand-alone HPGS is higher than that, for the utility connected HPGS. 
Table 6 the optimal results of utility connected HPGS at scenario II

\begin{tabular}{|c|c|c|c|}
\hline \multirow{2}{*}{ Variable } & \multicolumn{3}{|c|}{ Utility connected system (Scenario I) } \\
\cline { 2 - 4 } & MFO & MVO & FPA \\
\hline$A_{w}\left(m^{2}\right)$ & 4389 & 7381 & \\
\hline$A_{P V}\left(m^{2}\right)$ & 9546 & 8145 & \\
\hline$P_{b_{c a p}}(\mathrm{~kW})$ & 1000 & 1000 & 1000 \\
\hline$P_{b}(\mathrm{~kW})$ & 100 & 100 & 100 \\
\hline$P_{G T_{c a p}}(\mathrm{~kW})$ & 1500 & 1500 & 1500 \\
\hline$P_{U T_{c a p}}(\mathrm{~kW})$ & 212 & 233 & 232 \\
\hline$m \cdot\left(m^{3} / h r\right)$ & 413 & 408 & 426 \\
\hline TAC $(\$ /$ year $)$ & 563,401 & 524,536 & 572,098 \\
\hline SP (ton/year) & $2.0890 \mathrm{e}+11$ & $2.088 \mathrm{e}+11$ & $2.106 \mathrm{e}+11$ \\
\hline \multicolumn{4}{|l}{} \\
\hline
\end{tabular}

\section{CONCLUSION}

This paper presents a new contribution in the field of designing the HPGS, where the natural gas distribution network is included for the first time as a partner in the HPGS. The natural gas distribution network is involved in the HPGS to supply the fuel to the gas turbine. There two modes are discussed in this paper; the first one is a standalone and the second is utility connected mode. The HPGS in the standalone mode consists of WT, PV, SB, and GT. And it consists the same power sources to addition to the utility grid. All conditions of natural gas distribution network are studied and the most important parameters of this network are defined such as; the pressure drop and flow velocity. These parameters considered before the required amount of natural gas is delivered to the GT. The maximum allowable amount of the flow rate of the natural gas distribution network is calculated without exceeding the allowable limits of the pressure drop and flow velocity. Both of the standalone and utility connected is discussed for two different scenarios; winter and summer scenarios. There is a significant difference properly in all HPGS parameters through the two scenarios. For example, wind speed is $5.3(\mathrm{~m} / \mathrm{sec})$ in the winter season and it is $4.87(\mathrm{~m} / \mathrm{sec})$ in the summer season, the solar irradiance is $0.1005\left(\mathrm{~W} / \mathrm{m}^{2}\right)$ in the winter season while it is $0.1728\left(\mathrm{~W} / \mathrm{m}^{2}\right)$ in the summer season, and so on. MultiObjective functions have been design to optimally sizing the HPGS, and these functions are the TAC and SP. TAC consists the initial cost, operation and maintenance cost, salvage value, the annual fuel cost, and the annual purchasing electricity from the utility grid. SP presents the total annual emission (ton/year). All power system constraints and parameters are considered. The most two modern meta-heuristic optimization techniques are used to find the optimal sizing of the HPGS to find the minimum TAC and SP. MFO and MVO have been used to find the optimal multi-criteria design of the HPGS, where these techniques are used for the first time in designing the HPGS. a detailed comparison has been done for the designs of the different modes of the HPGS at different scenarios. Moreover, these techniques have been tested, where their results compared with the obtained from applying the FPA. The superiority and the effectiveness of using MFO and MVO could be observed and obtained.

\section{REFERENCES}

[1] L. Wang and C. Singh, "Multi-criteria Design of Hybrid Power Generation Systems Based on a Modified Particle Swarm Optimization Algorithm", IEEE Transactions on Energy Conversion, Vol. 24, no 1, 2009.

[2] L. Liqun and L. Chunxia, "Feasibility analyses of hybrid wind-PV-battery power system in Dongwangsha, Shanghai", PrzegladElektrotechniczny, Vol. 89, pp.239242, 2013.
[3] S. L. Trazouei, F. L. Tarazouei, and M. Ghiamy, "Optimal Design of a Hybrid Solar -Wind-Diesel Power System for Rural Electrification Using Imperialist Competitive Algorithm", International Journal of Renewable Energy Research, Vol.3, No.2, 2013.

[4] T. Tahri, A. Bettahar, and M. Douani, " Optimization of a Hybrid Wind-PV-Diesel Standalone System: Case Chlef, Algeria " International Journal of Mathematical, Computational, Physical and Quantum Engineering Vol.7, No.1, 2013.

[5] Q. A. J. Jawad1, K. K. Gasem, M. R. Jawad "Design and Simulation of Hybrid System for Electricity Generation in Iraqi Rural Regions", Diyala Journal of Engineering Sciences, Vol. 06, No. 02, pp. 38-56, 2013.

[6] T. Jima, "Simulation and Optimization of Wind Turbine, Solar PV, Storage Battery and Diesel Generator Hybrid Power System for a Cluster of Micro and Small Enterprises Working on Wood and Metal Products at Welenchity Site", M.Sc. Thesis, Addis Ababa Institute of Technology, 2013.

[7] A. Bhowmik, Design and scheduling of a Hybrid Power System consisting of Wind Turbine, PV Generator, Battery Bank and Diesel Generator using 'area based observe and focus' algorithm, M.Sc. Thesis, Jadavpur University, 2013.

[8] M. Sekar, S. Arunkumar, and V. Balasubramanian, "PVWind Hybrid Systems for Supplying Power to Remote Locations", International Journal of Advanced Technology \& Engineering Research (IJATER), ISSN No: 2250-3536, 2014.

[9] H. M. Farghally, F. H. Fahmy, and M. A. H.EL-Sayed, "Control and Optimal Sizing of PV-Wind Powered Rural Zone in Egypt", the Online Journal on Power and Energy Engineering (OJPEE), Vol. 2, No. 2, pp. 188-195, 2014.

[10] P. Gajbhiye, and P. Suhane, "Methodology for Optimal Sizing \& Power Management of Hybrid Energy System", International Journal of Electrical, Electronics and Computer Engineering, Vol. 03, No. 02, pp. 7-12, 2014

[11] H. Belmili, M. Haddadi, S. Bacha, M. F. Almi, "Sizing stand-alone photovoltaic-wind hybrid system: Technoeconomic analysis and optimization", Elsevier, Renewable and Sustainable Energy Reviews, Vol. 30, pp. 821-832, 2014.

[12] A.M. Eltamaly, and M. A. Mohamed, "A Novel Design and Optimization Software for Autonomous PV/Wind/Battery Hybrid Power Systems", International Journal of Photoenergy, Mathematical Problems in Engineering, Volume 2014, 2014.

[13] D. M. Atia, F. H. Fahmy, N. M. Ahmed, H. T. Dorrah, "Optimization and Feasibility Analysis of PV/Wind/ Battery Hybrid Energy Conversion" International Journal of Electrical, Robotics, Electronics and Communications Engineering Vol.8, No.5, 2014.

[14] A. Maleki, and A. Askarzadeh, " Optimum Configuration of Fuel Cell-Batteries PV/Wind Hybrid System using A Hybrid Meta Heuristic Technique", International Journal of Engineering \& Applied Sciences (IJEAS), Vol.5, Issue 4, pp.1-12, 2014.

[15] O. H. Mohammed, Y. Amirat, M. Benbouzid, and A.A. Elbaset, "Optimal Design of a PV/Fuel Cell Hybrid Power System for the City of Brest in France", IEEE ICGE, 2014. 
[16] M. S. Alam, "Optimal Enactment of a Stand-alone Hybrid Wind-Fuel Cell Based Distributed Generation System through Fuzzy Logic Control", BVICAM's International Journal of Information Technology, Vol. 6 No. 1, pp. 664-671, 2014.

[17] B. Tudu, K. K. MandaI, and N. Chakraborty Optimal Design and Performance Evaluation of A Grid Independent Hybrid Micro Hydro-Solar-Wind Fuel Cell Energy System Using Meta-Heuristic Techniques", IEEE, 1st International Conference on Non-Conventional Energy (ICONCE), 2014.
[18] Institute of Gas Engineers and Managers /Transmission and Distribution, Vol. 3, Edition 5, 2014.

[19] Gas Pressure Regulator Series 850 VARIFLO Catalog, RMG, 2010

[20] S. Mirjalili, " Moth-flame optimization algorithm: A Novel Nature-Inspired Heuristic Paradigm", Elsevier, Knowledge-Based Systems, 2015.

[21] S. Mirjalili, S. M. Mirjalili, and A. Hatamlou, " MultiVerse Optimizer: A Nature Inspired Algorithm for Global Optimization", Springer, Neural Comput \& Applic, 2015 